\title{
Inferences for Generalized Pareto Distribution Based on Progressive First-Failure Censoring Scheme
}

\author{
Rashad M. El-Sagheer, ${ }^{1}$ Taghreed M. Jawa, ${ }^{2}$ and Neveen Sayed-Ahmed $\mathbb{D}^{2}$ \\ ${ }^{1}$ Mathematics Department, Faculty of Science, Al-Azhar University, Nasr City 11884, Cairo, Egypt \\ ${ }^{2}$ Department of Mathematics and Statistics, College of Science, P.O. Box 11099, Taif University, Taif 21944, Saudi Arabia
}

Correspondence should be addressed to Neveen Sayed-Ahmed; nevensayd@yahoo.com

Received 22 October 2021; Accepted 15 November 2021; Published 7 December 2021

Academic Editor: Sameh S. Askar

Copyright (c) 2021 Rashad M. El-Sagheer et al. This is an open access article distributed under the Creative Commons Attribution License, which permits unrestricted use, distribution, and reproduction in any medium, provided the original work is properly cited.

\begin{abstract}
In this article, we consider estimation of the parameters of a generalized Pareto distribution and some lifetime indices such as those relating to reliability and hazard rate functions when the failure data are progressive first-failure censored. Both classical and Bayesian techniques are obtained. In the Bayesian framework, the point estimations of unknown parameters under both symmetric and asymmetric loss functions are discussed, after having been estimated using the conjugate gamma and discrete priors for the shape and scale parameters, respectively. In addition, both exact and approximate confidence intervals as well as the exact confidence region for the estimators are constructed. A practical example using a simulated data set is analyzed. Finally, the performance of Bayes estimates is compared with that of maximum likelihood estimates through a Monte Carlo simulation study.
\end{abstract}

\section{Introduction}

In life testing and reliability analysis, some units can be lost or withdrawn from the experiment before failure occurs. One of the major reasons for removal of the experimental units is to save the working experimental units for future use, thereby conserving the cost and time associated with testing. This leads us to use the censoring schemes. The type-II censoring can be considered a common type of censored scheme. Many authors have studied the statistical inference for different probability distributions using progressive type-II censoring, including Balakrishnan and Sandhu [1, 2], Cohen [3], Mann [4], Ng [5], Balakrishnan et al. [6], Gibbons and Vance [7], Yuen and Tse [8], Ng et al. [9], Balakrishnan [10], Soliman [11, 12], Madi and Raqab [13], Mahmoud et al. [14], Mahmoud et al. [15], Soliman et al. [16], El-Sagheer [17-19], Mahmoud et al. [20], ElSagheer and Hasaballah [21], El-Sagheer et al. [22], and Soliman et al. [23]. Recently, Zhang and Gui [24] studied the statistical inference for the lifetime performance index of Pareto distribution based on progressive type-II censored sample.
On the other hand, Viveros and Balakrishnan [25] have described a life test in which the experimenter can decide to divide the items being tested into several groups and then run all the items at the same time until occurrence of the first failure in each group. Such a censoring scheme is called first-failure censoring. For more details about statistical inference using first-failure censoring, it is recommended that the reader refers to $\mathrm{Wu}$ and $\mathrm{Yu}$ [26], Wu et al. [27], Lee et al. [28], and $\mathrm{Wu}$ et al. [29]. However, using this censoring scheme does not enable the experimenter to remove experimental units from the test until the first failure is observed. For this reason, $\mathrm{Wu}$ and Kuş [30] introduced a life testing scheme, which combines first-failure censoring with a progressive type-II censoring called a progressive first-failure censoring (Pro-F-F-C) scheme. Many previous studies have discussed inference under a Pro-F-F-C scheme for different lifetime distributions, for example, Weibull by $\mathrm{Wu}$ and Kuş [30], Burr Type XII by Soliman et al. [31, 32], Gompertz by Soliman et al. [33], Lomax by Mahmoud et al. [34], Compound Rayleigh by Abushal [35], Generalized Inverted Exponential by Ahmed [36], the Mixture of Weibull and Lomax 
by Mahmoud et al. [37], and exponentiated Frechet by Soliman et al. [38]. Recently, Cai and Gui [39] discussed the classical and Bayesian inference for a Pro-F-F-C lefttruncated normal distribution.

Generalized Pareto distribution (GPD) is a significant continuous lifetime distribution. It plays a key role in statistical inference studies and reliability problems. It is also well known for being a distribution that has decreasing failure rate property. The $\mathrm{pdf}$ and $\mathrm{cdf}$ of a random variable $X$ have a GPD given, respectively, as

$$
\begin{aligned}
& f_{X}(x ; \alpha, \beta)=\alpha \beta^{\alpha}(x+\beta)^{-(\alpha+1)}, x>0, \alpha, \beta>0, \\
& F_{X}(x ; \alpha, \beta)=1-\beta^{\alpha}(x+\beta)^{-\alpha}, x>0, \alpha, \beta>0,
\end{aligned}
$$

where $\alpha$ and $\beta$ are the shape and scale parameters, respectively. The survival and hazard rate functions of GPD at mission time $t$ are given by the following expressions:

$$
\begin{aligned}
& s(t)=\beta^{\alpha}(t+\beta)^{-\alpha}, t>0, \\
& h(t)=\alpha(t+\beta)^{-1}, \quad t>0 .
\end{aligned}
$$

For more details about GPD, its properties, and applications, see Kremer [40]. In this article, we obtain the Bayes estimates and MLEs for the unknown quantities of the GPD using a Pro-F-F-C scheme. The approximate confidence intervals (ACIs) for $\alpha$ and $\beta$ are constructed based on the asymptotic normality of MLEs. In the Bayesian framework, the point estimates of unknown parameters under squared error (SE), linear-exponential (LINEX), and general entropy (GE) showing loss functions are discussed. The process is done using the conjugate gamma prior for the shape parameter and discrete prior for the scale parameter $\beta$. The exact confidence interval and exact confidence region for the estimators are then derived. To evaluate and compare the performance of these proposed inference procedures, a simulation study with different parameter values is undertaken. Additionally, a numerical example using simulated data set is studied to show the practicality and usefulness of these proposed methods.

The rest of the paper is arranged as follows. Section 2 deals with the classical method of estimation. Bayes estimators relative to different loss functions are considered in Section 3. In Section 4, the ACIs, exact confidence intervals, and exact confidence regions for the parameters are discussed. In Section 5, the proposed procedures obtained in the previous sections are investigated using simulated data. A simulation study is conducted to compare the proposed procedures in Section 6. Finally, a conclusion is provided in Section 7.

\section{Maximum Likelihood Estimation}

Let $x_{i}=x_{i: m: n: k}^{R}, i=1,2, \ldots, m$, be a Pro-F-F-C order statistics from the GPD with the progressive censoring scheme $R=\left(R_{1}, R_{2}, \ldots, R_{m}\right)$. According to $\mathrm{Wu}$ and Kuş [30], the joint probability density function can be written as

$$
\begin{aligned}
& f_{1,2, \ldots, m}\left(x_{1: m: n: k}^{R}, \ldots, x_{m: m: n: k}^{R}\right) \propto k^{m} \coprod_{j=1}^{m} f\left(x_{j: m: n: k}^{R}\right) \\
& \quad\left(1-F\left(x_{j: m: n: k}^{R}\right)\right)^{k\left(R_{j}+1\right)-1} .
\end{aligned}
$$

From (1), (2), and (5), the likelihood function $L(\underline{x} ; \alpha, \beta)$ is given by

$$
L(\underline{x} ; \alpha, \beta) \propto k^{m} \alpha^{m} \prod_{i=1}^{m} \beta^{\alpha k\left(R_{i}+1\right)}\left(x_{i}+\beta\right)^{-\left(\alpha k\left(R_{i}+1\right)+1\right)} .
$$

Thus, the log-likelihood function $\ell(\underline{x} ; \alpha, \beta)$ is

$$
\begin{gathered}
\ell(\underline{x} ; \alpha, \beta) \propto m \log k+m \log \alpha+\sum_{i=1}^{m} \alpha k\left(R_{i}+1\right) \log \beta \\
-\sum_{i=1}^{m}\left(\alpha k\left(R_{i}+1\right)+1\right) \log \left(x_{i}+\beta\right) .
\end{gathered}
$$

By equating each result of the first-order derivatives of $\log$-likelihood function with respect to $\alpha$ and $\beta$, to zero, we obtain

$$
\begin{aligned}
\frac{\ell(\underline{x} ; \alpha, \beta)}{\partial \alpha}= & \frac{m}{\alpha}+\sum_{i=1}^{m} k\left(R_{i}+1\right) \log \beta \\
& -\sum_{i=1}^{m} k\left(R_{i}+1\right) \log \left(x_{i}+\beta\right)=0, \\
\frac{\ell(\underline{x} ; \alpha, \beta)}{\partial \beta}= & \sum_{i=1}^{m} \frac{\alpha k\left(R_{i}+1\right)}{\beta}-\sum_{i=1}^{m} \frac{\left(\alpha k\left(R_{i}+1\right)+1\right)}{\left(x_{i}+\beta\right)}=0 .
\end{aligned}
$$

Hence,

$$
\widehat{\alpha}=m\left(\sum_{i=1}^{m} k\left(R_{i}+1\right) \log \left(x_{i}+\widehat{\beta}\right)-\sum_{i=1}^{m} k\left(R_{i}+1\right) \log \widehat{\beta}\right)^{-1},
$$

and $\widehat{\beta}$ the solution of

$$
\sum_{i=1}^{m} \frac{\widehat{\alpha} k\left(R_{i}+1\right)}{\widehat{\beta}}-\sum_{i=1}^{m} \frac{\left(\widehat{\alpha} k\left(R_{i}+1\right)+1\right)}{\left(x_{i}+\widehat{\beta}\right)}=0 .
$$

Since there is no closed form of the solution to the above equations, the Newton-Raphson method (NRM) is widely used to obtain the desired MLEs in such situations. Once MLEs of $\alpha$ and $\beta$ are obtained, the MLEs of $s(t)$ and $h(t)$ for given $t$ can be obtained by the invariant property of the MLEs as

$$
\begin{aligned}
& \widehat{s}(t)=\widehat{\beta}^{\widehat{\alpha}}(t+\widehat{\beta})^{-\widehat{\alpha}}, t>0, \\
& \widehat{h}(t)=\widehat{\alpha}(t+\widehat{\beta})^{-1}, t>0 .
\end{aligned}
$$

\section{Bayesian Estimation}

Bayes estimation is quite different from the MLE method because it takes into consideration both the information from observed sample data and the prior information. Bayes' 
theorem is completely dependent on the parameter estimation through calculation of the posterior distribution. As calculating the posterior distribution is conditional on the data, this requires explicit specification of the prior distribution model parameters. Furthermore, in order to gain the best estimate of the unknown parameter, it is necessary to determine the appropriate loss functions.

The next step is to take into account different loss functions. First, we consider the square error (SE) loss function which is widely used in the literature. Because of the symmetry nature of this function, it gives equal weight to overestimation as well as underestimation. Under SE, the Bayesian estimate (BE) of any function of parameters, say $\psi(\Theta)=u(\alpha, \beta, s, h)$, is the unconditional posterior mean which is given as

$$
\widehat{\psi}_{B S}(\Theta)=E(\psi(\Theta))=\int_{\Theta} \psi(\Theta) \pi^{*}(\Theta) \mathrm{d} \Theta .
$$

However, in many situations, the parameter may be overestimated or show serious consequences of underestimation, or vice versa. In such cases, an asymmetric loss function, which associates greater importance to overestimation or underestimation, can be taken into consideration for parameters estimation. A beneficial asymmetric loss function is the LINEX loss as follows:

$$
L_{\operatorname{LINEX}}(\widehat{\psi}(\Theta), \psi(\Theta))=e^{a(\widehat{\psi}(\Theta)-\psi(\Theta))}-a(\widehat{\psi}(\Theta)-\psi(\Theta))-1,
$$

where $a$ is a shape parameter whose sign refers to the direction and its magnitude represents the degree of symmetry. Moreover, for $a$ figure close to zero, the LINEX loss more or less becomes a SE loss. Thus, the BE of $\psi(\Theta)$ under this loss function is given by

$$
\begin{aligned}
\widehat{\psi}_{B L}(\Theta) & =-\frac{1}{a} \log \left[E\left(e^{-a \psi(\Theta)}\right)\right] \\
& =-\frac{1}{a} \log \int_{\Theta} e^{-a \psi(\Theta)} \pi^{*}(\Theta) d \Theta .
\end{aligned}
$$

Next, we consider the GE loss function as follows:

$$
L_{G E}(\widehat{\psi}(\Theta), \psi(\Theta))=\left(\frac{\widehat{\psi}(\Theta)}{\psi(\Theta)}\right)^{q}-q \log \left(\frac{\widehat{\psi}(\Theta)}{\psi(\Theta)}\right)-1,
$$

where $q$ is a shape parameter which represents departure from symmetry. Subsequently, based on the GE loss functions, the $\mathrm{BE}$ of $\psi(\Theta)$ is obtained as

$$
\widehat{\psi}_{B G}(\Theta)=\left[E\left(\psi(\Theta)^{-q}\right)\right]^{-1 / q}=\left[\int_{\Theta}\left(\psi(\Theta)^{-q}\right) \pi^{*}(\Theta) \mathrm{d} \Theta\right]^{-1 / q} .
$$

It is remarked that for $q=-1$, the BE of $\psi(\Theta)$ concurs with the BE under SE loss function.
3.1. Posterior Analysis. In this subsection, we consider that the parameter $\beta$ a discrete prior and $\alpha$ has a conjugate gamma prior. Suppose that $\beta=\beta_{j}, j=1,2, \ldots, N$, then

$$
\pi(\beta)=\operatorname{Pr}\left(\beta=\beta_{j}\right)=\eta_{j},
$$

where $0 \leq \eta_{j} \leq 1$ and $\sum_{j=1}^{N} \eta_{j}=1$. Further, $\alpha$ has

$$
\pi\left(\alpha \mid \beta=\beta_{j}\right)=\frac{a_{j}^{b_{j}}}{\Gamma\left(b_{j}\right)} \alpha^{b_{j}-1} \exp \left(-a_{j} \alpha\right), \alpha ; a_{j}, b_{j}>0 .
$$
follows:

Then, the posterior distribution of $\alpha$ takes the form as

$$
\pi^{*}\left(\alpha \mid \beta=\beta_{j} ; T_{j}\right)=\frac{\left(T_{j}+a_{j}\right)^{\left(b_{j}+m\right)}}{\Gamma\left(b_{j}+m\right)} \alpha^{b_{j}+m-1} \exp \left(-\alpha\left(T_{j}+a_{j}\right)\right),
$$

where

$$
T_{j}=\sum_{i=1}^{m} k\left(R_{i}+1\right)\left[\log \left(x_{i}+\beta_{j}\right)-\log \beta_{j}\right] .
$$

The joint posterior of $\alpha$ and $\beta_{j}$ using (6), (19), and (20) is

$$
\pi^{*}\left(\alpha, \beta=\beta_{j}, T_{j}\right)=\frac{a_{j}^{b_{j}} v_{j} \eta_{j}}{k_{2} \Gamma\left(b_{j}\right)} \alpha^{b_{j}+m-1} \exp \left(-\alpha\left(T_{j}+a_{j}\right)\right),
$$

where

$$
\begin{aligned}
& k_{2}=\sum_{j=1}^{N} \Gamma \frac{a_{j}^{b_{j}} v_{j} \eta_{j} \Gamma\left(b_{j}+m\right)\left(T_{j}+a_{j}\right)^{\left(b_{j}+m\right)}}{} \\
& v_{j}=\prod_{i=1}^{m}\left(x_{i}+\beta_{j}\right)^{-1} .
\end{aligned}
$$

By using the Bayes theorem for discrete variables, the marginal posterior probability of $\beta$ is

$$
P_{j}=\operatorname{Pr}\left(\beta=\beta_{j} \mid T_{j}\right)=\frac{a_{j}^{b_{j}} v_{j} \eta_{j} \Gamma\left(b_{j}+m\right)}{k_{2} \Gamma\left(b_{j}\right)\left(T_{j}+a_{j}\right)^{\left(b_{j}+m\right)}},
$$

where $k_{2}$ and $v_{j}$ are given in (24); the marginal posterior probability of $\alpha$ is

$$
\pi^{*}\left(\alpha \mid T_{j}\right)=\sum_{j=1}^{m} \pi^{*}\left(\alpha \mid \beta=\beta_{j}, T_{j}\right) .
$$

3.2. BE under SE Loss. In this subsection, we obtain the BE of $\alpha, \beta, s(t)$, and $h(t)$ under SE loss function. By using (14), $(21)$, and (25), the BEs $\widetilde{\alpha}_{B S}, \widetilde{\beta}_{B S}, \widetilde{s}_{B S}(t)$, and $\widetilde{h}_{B S}(t)$ are given by 
$\widetilde{\alpha}_{B S}=\int_{0}^{\infty} \sum_{j=1}^{N} \alpha P_{j} \pi^{*}\left(\alpha \mid \beta=\beta_{j}, T_{j}\right) d \alpha=\sum_{j=1}^{N} P_{j} \frac{\left(b_{j}+m\right)}{\left(T_{j}+a_{j}\right)}, \widetilde{\beta}_{B S}=E_{\beta}(\beta \mid \underline{x})=\sum_{j=1}^{N} \beta_{j} P_{j}, \widetilde{s}_{B S}(t)=\sum_{j=1}^{N} P_{j}\left[1+\frac{\log \left(1+\left(t / \beta_{j}\right)\right)}{\left(T_{j}+a_{j}\right)}\right]^{\left(b_{j}+m\right)}$,

$\widetilde{h}_{B S}(t)=\sum_{j=1}^{N} \frac{P_{j}\left(b_{j}+m\right)}{\left(t+\beta_{j}\right)\left(T_{j}+a_{j}\right)}$.

3.3. BE under LINEX Loss. Based on (16), (21), and (25), the BEs $\widetilde{\beta}_{B L}, \widetilde{\alpha}_{B L}, \widetilde{s}_{B L}(t)$, and $\widetilde{h}_{B L}(t)$ are

$$
\begin{aligned}
\tilde{\beta}_{B L} & =-\frac{1}{a} \log \left[\sum_{j=1}^{N} P_{j} \exp \left(-a \beta_{j}\right)\right], \\
\widetilde{\alpha}_{B L} & =-\frac{1}{a} \log \left[\sum_{j=1}^{N} P_{j}\left(1+\frac{a}{\left(T_{j}+a_{j}\right)}\right)^{-\left(b_{j}+m\right)}\right], \\
\widetilde{s}_{B L}(t) & =-\frac{1}{a} \log \left[\sum_{j=1}^{N} \sum_{\varepsilon=1}^{\infty} \frac{(-a)^{\varepsilon}}{\varepsilon !} P_{j}\left(1+\frac{\varepsilon \log \left(1+t / \beta_{j}\right)}{\left(T_{j}+a_{j}\right)}\right)^{-\left(b_{j}+m\right)}\right], \\
\widetilde{h}_{B L}(t) & =-\frac{1}{a} \log \left[\sum_{j=1}^{N} P_{j}\left(1+\frac{a}{\left(t+\beta_{j}\right)\left(T_{j}+a_{j}\right)}\right)^{-\left(b_{j}+m\right)}\right] .
\end{aligned}
$$

3.4. BE under GE Loss. From (18), (21), and (25), the BEs $\widetilde{\beta}_{B G}$, $\widetilde{\alpha}_{B G}, \widetilde{s}_{B G}(t)$, and $\widetilde{h}_{B G}(t)$ are, respectively,

$$
\begin{gathered}
\tilde{\beta}_{B G}=\left[\sum_{j=1}^{N} \beta_{j}^{-q} P_{j}\right]^{(-1 / q)}, \\
\tilde{\alpha}_{B G}=\left[\sum_{j=1}^{N} P_{j} \frac{\left(T_{j}+a_{j}\right)^{q} \Gamma\left(b_{j}+m-q\right)}{\Gamma\left(b_{j}+m\right)}\right]^{(-1 / q)}, \\
\widetilde{s}_{B G}(t)=\left[\sum_{j=1}^{N} P_{j}\left(1-\frac{q \log \left(1+\left(t / \beta_{j}\right)\right)}{\left(T_{j}+a_{j}\right)}\right)^{-\left(b_{j}+m\right)}\right]^{(-1 / q)}, \\
\tilde{h}_{B G}(t)=\left[\sum_{j=1}^{N} P_{j}\left(t+\beta_{j}\right)^{q} \times \frac{\left(T_{j}+a_{j}\right)^{q} \Gamma\left(b_{j}+m-q\right)}{\Gamma\left(b_{j}+m\right)}\right]^{(-1 / q)} .
\end{gathered}
$$

To perform the calculations in these subsections, the values of $a_{j}$ and $b_{j}$ must be found in (20). We use the prior expectation of $s(t)$ conditional on $\beta=\beta_{j}$. Thus, from (3) and (20), we get

$$
E\left[s(t) \mid \beta_{j}\right]=\left(1+\frac{\log \left(1+\left(t / \beta_{j}\right)\right)}{a_{j}}\right)^{b_{j}}
$$

\section{Interval Estimation}

This section deals with ACIs, exact CIs, and exact confidence regions for the parameters $\alpha$ and $\beta$ of GPD based on Pro-FF-C.

4.1. Asymptotic Confidence Intervals. The asymptotic normality of the MLEs can be used to construct ACIs for parameters $\alpha$ and $\beta$ by using Fisher information matrix (FIM). The FIM can be written as $I=\left(I_{i j}\right)$ where

$$
I_{i j}=E\left[\frac{-\partial^{2} \ell(\Phi)}{\partial \phi_{i} \partial \phi_{j}}\right], \quad i, j=1,2
$$

where $\Phi=\left(\phi_{1}, \phi_{2}\right)=(\alpha, \beta)$. The asymptotic variance-covariance matrix of the parameters $\alpha$ and $\beta$ can be obtained by inverting the observed FIM $I_{i j}$ as follows:

$$
I^{-1}(\widehat{\alpha}, \widehat{\beta})=\left[\begin{array}{c}
-\frac{\partial^{2} \ell}{\partial \alpha^{2}}-\frac{\partial^{2} \ell}{\partial \alpha \partial \beta} \\
-\frac{\partial^{2} \ell}{\partial \beta \partial \alpha}-\frac{\partial^{2} \ell}{\partial \beta^{2}}
\end{array}\right]_{(\widehat{\alpha}, \widehat{\beta})}^{-1}=\left[\begin{array}{c}
\operatorname{var}(\widehat{\alpha}) \operatorname{cov}(\widehat{\alpha}, \widehat{\beta}) \\
\operatorname{cov}(\widehat{\beta}, \widehat{\alpha}) \operatorname{var}(\widehat{\beta})
\end{array}\right],
$$

with

$$
\begin{aligned}
\frac{\partial^{2} \ell}{\partial \alpha^{2}} & =-\frac{m}{\alpha^{2}} \\
\frac{\partial^{2} \ell}{\partial \alpha \partial \beta} & =\frac{\partial^{2} \ell}{\partial \beta \partial \alpha}=\sum_{i=1}^{m} \frac{k\left(R_{i}+1\right)}{\beta}-\sum_{i=1}^{m} \frac{k\left(R_{i}+1\right)}{\left(x_{i}+\beta\right)}, \\
\frac{\partial^{2} \ell}{\partial \beta^{2}} & =\sum_{i=1}^{m} \frac{\alpha k\left(R_{i}+1\right)+1}{\left(x_{i}+\beta\right)^{2}}-\sum_{i=1}^{m} \frac{\alpha k\left(R_{i}+1\right)}{\beta^{2}} .
\end{aligned}
$$

Thus,

$$
(\widehat{\alpha}, \widehat{\beta}) \sim N\left((\alpha, \beta), I_{0}^{-1}(\widehat{\alpha}, \widehat{\beta})\right)
$$

The $(1-\delta) 100 \%$ ACIs for $\alpha$ and $\beta$ become 


$$
\begin{aligned}
& \left(\widehat{\alpha}-Z_{\delta / 2} \sqrt{\operatorname{var}(\widehat{\alpha})}, \widehat{\alpha}+Z_{\delta / 2} \sqrt{\operatorname{var}(\widehat{\alpha})}\right), \\
& \quad\left(\widehat{\beta}-Z_{\delta / 2} \sqrt{\operatorname{var}(\widehat{\beta})}, \widehat{\beta}+Z_{\delta / 2} \sqrt{\operatorname{var}(\widehat{\beta})}\right),
\end{aligned}
$$

where $z_{\delta}$ is $100(1-\delta)$ th upper percentile of standard normal variate $N(0,1)$.

4.2. Exact Confidence Intervals. Let $x_{1: m: n: k}^{\mathbf{R}}<x_{2: m: n: k}^{\mathbf{R}}<$ $\cdots<x_{m: m: n: k}^{\mathbf{R}}$ denote a Pro-F-F-C sample from GPD with parameters $\alpha$ and $\beta$, and let

$$
U_{i: m: n: k}^{R}=k \alpha \log \left(1+\frac{x_{i: m: n: k}^{R}}{\beta}\right), \quad i=1,2, \ldots, m .
$$

It is remarked that $U_{1: m: n: k}^{R}<U_{2: m: n: k}^{R}<\ldots<$ $U_{m: m: n: k}^{R}$ is a progressively censored sample of exponential distribution (ED) with mean 1. Let us assume the following:

$$
\left\{\begin{array}{l}
W_{1}=n U_{1: m: n: k}^{\mathbf{R}} \\
W_{2}=\left(n-R_{1}-1\right)\left(U_{2: m: n: k}^{\mathbf{R}}-U_{1: m: n: k}^{\mathbf{R}}\right) \\
W_{3}=\left(n-R_{1}-R_{2}-2\right)\left(U_{3: m: n: k}^{\mathbf{R}}-U_{2: m: n: k}^{\mathbf{R}}\right) \\
\vdots \\
W_{m}=\left(n-R_{1}-\ldots-R_{m-1}-m+1\right)\left(U_{m: m: n: k}^{\mathbf{R}}-U_{m-1: m: n: k}^{\mathbf{R}}\right)
\end{array}\right.
$$

According to Thomas and Wilson [41], the generalized spacings $W_{1}, W_{2}, \ldots, W_{m}$ are iid as standard ED; hence,

$$
\zeta_{j}=2 \sum_{i=1}^{j} W_{i}
$$

$$
\psi_{j}=2 \sum_{i=j+1}^{m} W_{i}
$$

has $X^{2} 2(m-j)$. To construct the confidence intervals for $\alpha$ and $\beta$, we consider pivotal quantities:

has $X^{2}(2 j)$, and

$$
\begin{gathered}
\xi_{j}=\frac{\psi_{j} /(2(m-j))}{\zeta_{j} / 2 j}=\frac{j}{(m-j)} \cdot \frac{2 \sum_{i=j+1}^{m} W_{i}}{2 \sum_{i=1}^{j} W_{i}} \\
=\frac{j}{(m-j)} \cdot \frac{\left(R_{1}+R_{2}+\cdots+R_{j}+j-n\right)+\sum_{i=j+1}^{m}\left(R_{i}+1\right) \log \left(1+\left(x_{i: m: n: k}^{R} / \beta\right)\right) / \log \left(1+\left(x_{j: m: n: k}^{R} / \beta\right)\right)}{\left(n-R_{1}-R_{2}-\ldots-R_{j-1}-j+1\right)+\sum_{i=1}^{j-1}\left(R_{i}+1\right) \log \left(1+\left(x_{i: m: n: k}^{R} / \beta\right)\right) / \log \left(1+\left(x_{j: m: n: k}^{R} / \beta\right)\right)} \quad j=1,2, \ldots, m-1, \\
\begin{aligned}
\eta & =\left(\psi_{j}+\zeta_{j}\right)=2 \sum_{i=1}^{m} W_{i}=2 \sum_{i=1}^{m}\left(R_{i}+1\right) U_{i: m: n: k}^{R} \\
& =2 k \alpha \sum_{i=1}^{m}\left(R_{i}+1\right) \log \left(1+\frac{\left.x_{i: m: n: k}^{R}\right)}{\beta}\right)
\end{aligned}
\end{gathered}
$$

It can be easily shown that $\xi_{j} \sim F(2(m-j), 2 j)$ where $j=1,2, \ldots, m-1, m>1$, and $\eta \sim X^{2}(2 m)$. Also, $\xi_{j}$ and $\eta$ are independent. To construct an exact confidence interval for $\beta$ and exact joint confidence region for $\beta$ and $\alpha$, we need to analyze the following two lemmas.

Lemma 1. For any positive real numbers $b>a>0, q(\gamma)=$ $\ln \left(1+b^{\gamma}\right) / \ln \left(1+a^{\gamma}\right)$ is a strictly increasing function of $\gamma$, where $\gamma>0$.
Lemma 2. For a given set of observations $0<x_{1: m: n: k}^{\mathbf{R}}<$ $x_{2: m: n: k}^{\mathbf{R}}<\ldots<x_{m: m: n: k}^{\mathbf{R}}<\infty$, the function $\xi_{j}$ is a strictly increasing function of $\beta$ when $\beta>0$. Furthermore,

(I) For $x_{m-1: m: n: k}^{\mathbf{R}} \leq 1$, there is a unique solution for the given equation $\xi_{j}=t$, where $t>0$.

(II) Let $x_{0: m: n: k}^{\mathbf{R}}=0$. For $x_{l: m: n: k}^{\mathbf{R}} \leq 1<x_{l+1: m: n: k}^{\mathbf{R}}$, there is a unique solution for the given equation $\xi_{j}=t$ where 


$$
0<t<\frac{j}{(m-j)} \cdot \frac{\sum_{i=j+1}^{m}\left(R_{i}+1\right) \log \left(x_{i: m: n: k}^{R}\right)-\left(n-R_{1}-R_{2}-\ldots-R_{j}-j\right) \log \left(x_{j: m: n: k}^{R}\right)}{\left(n-R_{1}-R_{2}-\ldots-R_{j-1}-j+1\right) \log \left(x_{j: m: n: k}^{R}\right)+\sum_{i=l+1}^{j-1}\left(R_{i}+1\right) \log \left(x_{i: m: n: k}^{R}\right)},
$$

for $l=0,1, \ldots, j-1$ and $j=1,2, \ldots, m-1$. Using the same arguments and notations in $W u$ et al. [42], Lemma 1 and Lemma 2 can be proved.

4.3. Exact Confidence Interval for $\beta$. Suppose that $x_{i, m, n, k}^{\mathbf{R}}$, $i=1,2, \ldots, m$, denote a Pro-F-F-C sample from GPD $(\alpha, \beta)$, with censoring scheme $\left(R_{1}, R_{2}, \ldots, R_{m}\right)$. For any $0<\delta<1$, $a 100(1-\delta) \%$ confidence interval for $\beta$ is as follows. We know that $\xi_{j} \sim F_{(2(m-j), 2 j)}$ by Lemma1 and Lemma $2 \xi_{j}$ strictly increases in $\beta$ when $\beta>0$, where

(1) For $x_{m-1: m: n: k}^{\mathbf{R}} \leq 1$, there is a unique solution for the given equation $\xi_{j}=t$, where $t>0$.
(2) Let $x_{0: m: n: k}^{\mathbf{R}}=0$. For $x_{l: m: n: k}^{\mathbf{R}} \leq 1<x_{l+1: m: n: k}^{\mathbf{R}}$, there is a unique solution for the equation $\xi_{j}=t$.

Hence, for $0<\delta<1$, from (44), we obtain

$$
F_{1-\frac{\delta}{2}(2(m-j), 2 j)}<\xi_{j}<F_{\delta}(2(m-j), 2 j) .
$$

Thus, a $100(1-\delta) \%$ confidence interval for $\beta$ is

$$
\left(\Phi\left(X^{R}, F_{1-\delta / 2(2(m-j), 2 j)}\right)<\beta<\Phi\left(X^{R}, F_{\delta / 2(2(m-j), 2 j)}\right)\right),
$$

where $\quad X^{R}=\left(X_{1: m: n: k}^{R}, X_{2: m: n: k}^{R}, \ldots, X_{m: m: n: k}^{R}\right) \quad$ and $\Phi\left(X^{R}, t\right)$ is the solution for $\beta$ for the equation:

$$
\frac{\left(R_{1}+R_{2}+\cdots+R_{j}+j-n\right)+\sum_{i=j+1}^{m}\left(R_{i}+1\right) \log \left(1+x_{i: m: n: k}^{R} / \beta\right) / \log \left(1+x_{j: m: n: k}^{R} / \beta\right)}{\left(n-R_{1}-R_{2}-\ldots-R_{j-1}-j+1\right)+\sum_{i=1}^{j-1}\left(R_{i}+1\right) \log \left(1+x_{i: m: n: k}^{R} / \beta\right) / \log \left(1+x_{j: m: n: k}^{R} / \beta\right)}=\frac{t(m-j)}{j} .
$$

4.4. Exact Confidence Region for $\beta$ and $\alpha$. By the same way,

$$
P\left(F_{(1+\sqrt{1-\delta} / 2 / 2)(2(m-j), 2 j)}<\xi_{j}<F_{1-\sqrt{1-\delta} / 2(2(m-j), 2 j)}\right)=\sqrt{1-\delta},
$$

$$
\eta=2 k \alpha \sum_{i=1}^{m}\left(R_{i}+1\right) \log \left(1+\frac{x_{i: m: n: k}^{R}}{\beta}\right)
$$

where $\eta \sim X^{2}(2 m)$. For $0<\delta<1$, we have

$$
P\left(\chi_{1+\sqrt{1-\delta} / 2(2 m)}^{2}<\eta<\chi_{1-\sqrt{1-\delta} / 2(2 m)}^{2}\right)=\sqrt{1-\delta} .
$$

Then, we obtain

$$
\begin{array}{r}
P\left(F_{1+\sqrt{1-\delta} / 2(2(m-j), 2 j)}<\xi_{j}<F_{1-\sqrt{1-\delta} / 2(2(m-j), 2 j)},\right. \\
\left.\chi_{1+\sqrt{1-\delta} / 2(2 m)}^{2}<\eta<\chi_{1+\sqrt{1-\delta} / 2(2 m)}^{2}\right)=1-\delta .
\end{array}
$$

This is equivalent to

$$
\begin{aligned}
& P\left(\Phi\left(X^{R}, F_{1+\sqrt{1-\delta} / 2(2(m-j), 2 j)}\right)<\beta<\Phi\left(X^{R}, F_{1-\sqrt{1-\delta} / 2(2(m-j), 2 j)}\right), \frac{\chi_{1+\sqrt{1-\delta} / 2(2 m)}^{2}}{2 k \sum_{i=1}^{m}\left(R_{i}+1\right) \log \left(1+x_{i: m: n: k}^{R} / \beta\right)}\right. \\
& \left.\quad<\alpha<\frac{\chi_{1-\sqrt{1-\delta} / 2(2 m)}^{2}}{2 k \sum_{i=1}^{m}\left(R_{i}+1\right) \log \left(1+x_{i: m: n: k}^{R} / \beta\right)}\right)=1-\delta .
\end{aligned}
$$

\section{Numerical Computations}

Consider a Pro-F-F-C sample generated from GPD showing $\alpha=0.3$ and $\beta=1.5$. The data consist of 120 observations, grouped into $n=30$ sets, with 4 items within each group $(k=4)$. The Pro-F-F-C sample of size 10 out of 30 groups with the corresponding censoring scheme $R$ is given in Table 1 . The MLEs of $\alpha$ and $\beta$ using NRM are computed, and then both $s(t)$ and $h(t)$ are calculated at $t=0.451$.

To compute the BEs, we first estimate two values of $s(t)$ using a nonparametric procedure $s\left(t_{i}=x_{i, m, n, k}^{R}\right)=m-i+$ $0.625 / m+0.25, i=1,2, \ldots, m$. Using the available data, we obtained $s\left(t_{1}=0.1694\right)=0.7439$ and $s\left(t_{2}=4.8110\right)=$ 0.1585 . These two priors are substituted into (33), where $a_{j}$ and $b_{j}$ are obtained numerically for each given $\beta_{j}$, and $\eta_{j}$, $j=1,2, \ldots, 10$, using the NRM. Table 2 displays the values of $a_{j}, b_{j}$, and $P_{j}$ for each given $\beta_{j}$ and $\eta_{j}$. The results of MLE and BE for $\alpha, \beta, s(t)$, and $h(t)$ are presented in Table 3. By using (45), the 95\% ACIs of $\alpha$ and $\beta$ are $(0,0.7068)$ and $(0,4.4543)$. For $j=2$, we need the percentiles $F_{0.025}(18,2)=$ 0.2193 and $F_{0.975}(18,2)=39.4424$ to construct the $95 \% \mathrm{CI}$ for $\beta$. According to (44), the $95 \%$ exact confidence interval of $\beta$ is calculated as $(0.2193,7.9943)$. For the given $F_{0.0127}$ $(18,2)=0.1780, \quad F_{0.9873}(18,2)=78.1835, \quad \chi_{(0.0127)(20)}^{2}=$ 
Table 1: Simulated Pro-F-F-C.

\begin{tabular}{lcccccccccc}
\hline$i$ & 1 & 2 & 3 & 4 & 5 & 6 & 7 & 8 & 9 & 10 \\
\hline$R_{i}$ & 10 & 0 & 1 & 1 & 5 & 1 & 1 & 1 & 0 & 0 \\
$x_{i}^{R}$ & 0.0781 & 0.1582 & 0.1694 & 0.2040 & 0.3066 & 0.4909 & 0.8912 & 1.0705 & 4.811 & 14.123 \\
\hline
\end{tabular}

TABLE 2: The hyperparameter values.

\begin{tabular}{ccccccccccc}
\hline$j$ & 1 & 2 & 3 & 4 & 5 & 6 & 7 & 8 & 9 & 10 \\
\hline$\eta_{j}$ & 0.1 & 0.1 & 0.1 & 0.1 & 0.1 & 0.1 & 0.1 & 0.1 & 0.1 & 0.1 \\
$\beta_{j}$ & 1.0 & 1.1 & 1.2 & 1.3 & 1.4 & 1.5 & 1.6 & 1.7 & 1.8 & 1.9 \\
$a_{j}$ & 0.6995 & 0.5966 & 0.5184 & 0.4571 & 0.4087 & 0.3684 & 0.3349 & 0.3066 & 0.2824 & 0.2615 \\
$b_{j}$ & 1.4647 & 1.3743 & 1.3054 & 1.2457 & 1.1976 & 1.1574 & 1.1237 & 1.0938 & 1.0664 & 1.0434 \\
$P_{j}$ & 0.1059 & 0.1058 & 0.1085 & 0.1052 & 0.1041 & 0.1023 & 0.0997 & 0.0961 & 0.0921 & 0.0857 \\
\hline
\end{tabular}

TABLE 3: The MLEs and BEs of $\alpha, \beta, s(t)$, and $h(t)$ where $s(0.450)=0.9243$ and $h(0.450)=0.1538$.

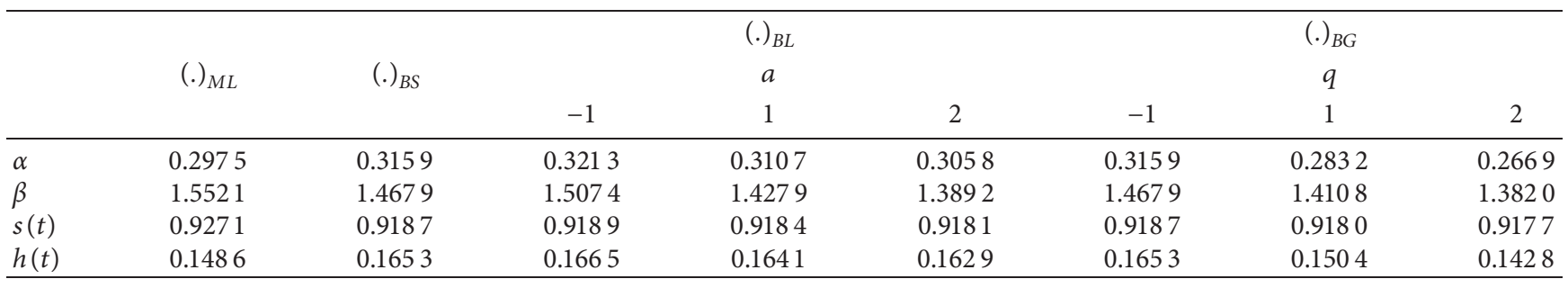

Table 4: The interval lengths for $\beta$ and 95\% confidence area for $\alpha$ and $\beta$.

\begin{tabular}{lcr}
\hline$j$ & Length & Area \\
\hline 1 & 7.3419 & 198.238 \\
2 & 6.1107 & 74.2141 \\
3 & 5.7796 & 61.2357 \\
4 & 4.3371 & 66.9821 \\
5 & 4.7508 & 64.8714 \\
6 & 4.2892 & 59.4761 \\
7 & 4.2547 & 55.2478 \\
8 & 4.0687 & 62.4790 \\
9 & 4.3541 & 67.2178 \\
10 & 5.1017 & 55.4785 \\
11 & 5.1899 & 61.2587 \\
12 & 4.6457 & 56.4512 \\
13 & 5.2475 & 42.8979 \\
14 & 4.5626 & 39.4872 \\
15 & 6.9847 & 41.2789 \\
\hline
\end{tabular}

8.5737, and $\chi_{(0.9873)(20)}^{2}=36.7141$, the $95 \%$ joint confidence region for $\beta$ and $\alpha$ is

$$
\left\{\begin{array}{l}
0.2193<\beta<709943 \\
\frac{8.5737}{8 \sum_{i=1}^{m}\left(R_{i}+1\right) \log \left(1+x_{i: m: n: k}^{R} / \beta\right)}<\alpha \\
<\frac{36.7141}{8 \sum_{i=1}^{m}\left(R_{i}+1\right) \log \left(1+x_{i: m: n: k}^{R} / \beta\right)}
\end{array}\right.
$$

After the following integration,

$$
\int_{0.2193}^{7.9943} \frac{34.5235}{2 k \sum_{i=1}^{m}\left(R_{i}+1\right) \log \left(1+\left(x_{i: m: n: k}^{R} / \beta\right)\right)} \mathrm{d} \beta .
$$

We obtain the confidence area at $j=2$, by 74.2141 . Similarly, the confidence areas for some values of $j$ are presented in Table 4. Figure 1 shows the 95\% confidence region for $\beta$ and $\alpha$.

\section{Simulation Study}

To compare the proposed BEs with the MLEs, a simulation study is performed using various combinations of $n, m$, and $k$ and different censored schemes of $R$ (different $R_{i}$ values). A 


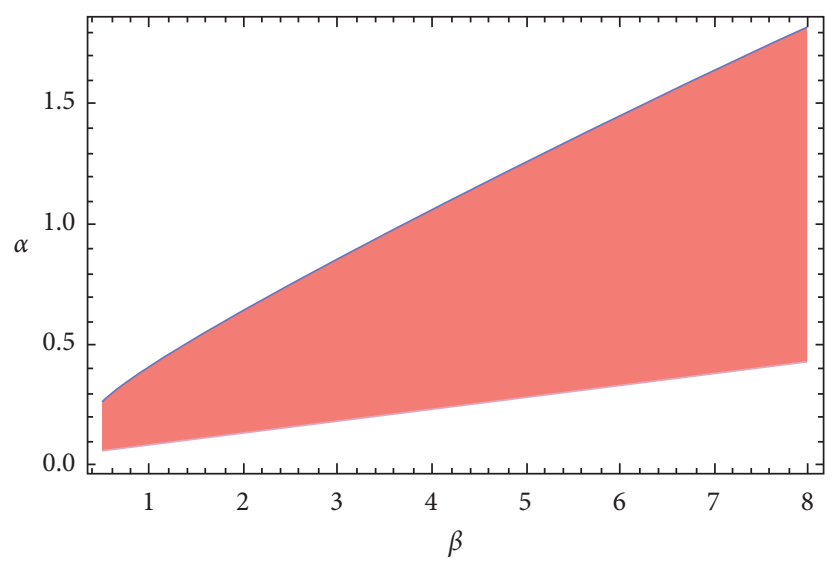

Figure 1: Joint confidence region for $\beta$ and $\alpha$.

TABLe 5: MSE of MLEs and BEs with true values.

\begin{tabular}{|c|c|c|c|c|c|c|c|c|c|c|}
\hline \multirow{3}{*}{$k$} & \multirow{3}{*}{$n$} & \multirow{3}{*}{$m$} & \multirow{3}{*}{ C.S } & & \multirow{3}{*}{$M L$} & \multirow{3}{*}{ BS } & \multirow{2}{*}{\multicolumn{2}{|c|}{$\begin{array}{c}B L \\
a\end{array}$}} & \multirow{2}{*}{\multicolumn{2}{|c|}{$\begin{array}{c}B G \\
q\end{array}$}} \\
\hline & & & & & & & & & & \\
\hline & & & & & & & -1 & 1 & -1 & 1 \\
\hline \multirow{12}{*}{1} & \multirow{12}{*}{30} & \multirow{12}{*}{20} & \multirow{4}{*}{ I } & $\tilde{\alpha}$ & 0.1576 & 0.0920 & 0.0921 & 0.0920 & 0.0920 & 0.0919 \\
\hline & & & & $\widetilde{\beta}$ & 0.7884 & 0.5711 & 0.5744 & 0.5669 & 0.5711 & 0.5655 \\
\hline & & & & $\widetilde{\widetilde{s}}(t)$ & 0.0256 & 0.0223 & 0.0229 & 0.0223 & 0.0223 & 0.0216 \\
\hline & & & & $\widetilde{h}(t)$ & 0.0056 & 0.0042 & 0.0042 & 0.0041 & 0.0042 & 0.0040 \\
\hline & & & \multirow{4}{*}{ II } & $\tilde{\alpha}$ & 0.1699 & 0.0922 & 0.0922 & 0.0921 & 0.0922 & 0.0919 \\
\hline & & & & $\widetilde{\beta}$ & 0.8268 & 0.5735 & 0.5770 & 0.5680 & 0.5735 & 0.5584 \\
\hline & & & & $\underset{\sim}{\tilde{s}}(t)$ & 0.0258 & 0.0236 & 0.0236 & 0.0231 & 0.0236 & 0.0221 \\
\hline & & & & $\tilde{h}(t)$ & 0.0058 & 0.0044 & 0.0044 & 0.0042 & 0.0044 & 0.0041 \\
\hline & & & \multirow{4}{*}{ III } & $\tilde{\alpha}$ & 0.1801 & 0.0956 & 0.0957 & 0.0939 & 0.0956 & 0.0938 \\
\hline & & & & $\tilde{\beta}$ & 0.8314 & 0.5788 & 0.5789 & 0.5695 & 0.5788 & 0.5595 \\
\hline & & & & $\underset{\sim}{\tilde{s}}(t)$ & 0.0259 & 0.0237 & 0.0239 & 0.0235 & 0.0237 & 0.0227 \\
\hline & & & & $\widetilde{h}(t)$ & 0.0061 & 0.0046 & 0.0048 & 0.0045 & 0.0046 & 0.0043 \\
\hline \multirow{12}{*}{5} & \multirow{12}{*}{30} & \multirow{12}{*}{20} & \multirow{4}{*}{ I } & $\underset{\sim}{\tilde{\alpha}}$ & 0.1588 & 0.0951 & 0.0952 & 0.0948 & 0.0951 & 0.0944 \\
\hline & & & & $\tilde{\beta}$ & 0.6453 & 0.5717 & 0.5748 & 0.5673 & 0.5717 & 0.5663 \\
\hline & & & & $\widetilde{\boldsymbol{s}}(t)$ & 0.0160 & 0.0151 & 0.0149 & 0.0147 & 0.0151 & 0.0146 \\
\hline & & & & $\widetilde{h}(t)$ & 0.0049 & 0.0041 & 0.0042 & 0.0040 & 0.0041 & 0.0039 \\
\hline & & & \multirow{4}{*}{ II } & $\underset{\tilde{\alpha}}{\sim}$ & 0.1597 & 0.0967 & 0.0965 & 0.0963 & 0.0967 & 0.0953 \\
\hline & & & & $\widetilde{\beta}$ & 0.6680 & 0.5724 & 0.5751 & 0.5682 & 0.5724 & 0.5674 \\
\hline & & & & $\widetilde{\widetilde{s}}(t)$ & 0.0168 & 0.0152 & 0.0153 & 0.0149 & 0.0152 & 0.0148 \\
\hline & & & & $\tilde{h}(t)$ & 0.0052 & 0.0046 & 0.0049 & 0.0045 & 0.0046 & 0.0043 \\
\hline & & & \multirow{4}{*}{ III } & $\underset{\widetilde{\alpha}}{\sim}$ & 0.1602 & 0.0969 & 0.0976 & 0.0968 & 0.0969 & 0.0957 \\
\hline & & & & $\widetilde{\beta}$ & 0.6692 & 0.5755 & 0.5783 & 0.5684 & 0.5755 & 0.5683 \\
\hline & & & & $\underset{\sim}{\widetilde{s}}(t)$ & 0.0175 & 0.0157 & 0.0158 & 0.0155 & 0.0157 & 0.0152 \\
\hline & & & & $\widetilde{h}(t)$ & 0.0057 & 0.0047 & 0.0051 & 0.0048 & 0.0047 & 0.0045 \\
\hline \multirow{12}{*}{1} & \multirow{12}{*}{30} & \multirow{12}{*}{25} & \multirow{4}{*}{ I } & $\underset{\sim}{\tilde{\alpha}}$ & 0.1501 & 0.0886 & 0.0889 & 0.0885 & 0.0886 & 0.0881 \\
\hline & & & & $\tilde{\beta}$ & 0.7861 & 0.5679 & 0.5680 & 0.5593 & 0.5679 & 0.5275 \\
\hline & & & & $\widetilde{\boldsymbol{s}}(t)$ & 0.0254 & 0.0218 & 0.0222 & 0.0217 & 0.0218 & 0.0215 \\
\hline & & & & $\widetilde{h}(t)$ & 0.0053 & 0.0040 & 0.0041 & 0.0039 & 0.0040 & 0.0036 \\
\hline & & & & $\widetilde{\alpha}$ & 0.1524 & 0.0897 & 0.0898 & 0.0889 & 0.0897 & 0.0886 \\
\hline & & & & $\widetilde{\beta}$ & 0.7952 & 0.5683 & 0.5685 & 0.5596 & 0.5683 & 0.5297 \\
\hline & & & II & $\underset{\sim}{\widetilde{S}}(t)$ & 0.0259 & 0.0231 & 0.0239 & 0.0229 & 0.0231 & 0.0227 \\
\hline & & & & $\widetilde{h}(t)$ & 0.0054 & 0.0041 & 0.0042 & 0.0040 & 0.0041 & 0.0038 \\
\hline & & & & $\underset{\tilde{\alpha}}{\sim}$ & 0.1537 & 0.0898 & 0.0899 & 0.0892 & 0.0898 & 0.0889 \\
\hline & & & & $\tilde{\beta}$ & 0.7959 & 0.5692 & 0.5695 & 0.5608 & 0.5692 & 0.5343 \\
\hline & & & III & $\widetilde{\mathcal{S}}(t)$ & 0.0266 & 0.0246 & 0.0248 & 0.0245 & 0.0246 & 0.0239 \\
\hline & & & & $\widetilde{h}(t)$ & 0.0055 & 0.0045 & 0.0048 & 0.0044 & 0.0045 & 0.0041 \\
\hline
\end{tabular}


TABle 5: Continued.

\begin{tabular}{|c|c|c|c|c|c|c|c|c|c|c|}
\hline \multirow{3}{*}{$k$} & \multirow{3}{*}{$n$} & \multirow{3}{*}{$m$} & \multirow{3}{*}{ C.S } & & \multirow{3}{*}{$M L$} & \multirow{3}{*}{$B S$} & \multirow{2}{*}{\multicolumn{2}{|c|}{$\begin{array}{c}B L \\
a\end{array}$}} & \multirow{2}{*}{\multicolumn{2}{|c|}{$\begin{array}{c}B G \\
q\end{array}$}} \\
\hline & & & & & & & & & & \\
\hline & & & & & & & -1 & 1 & -1 & 1 \\
\hline \multirow{12}{*}{5} & \multirow{12}{*}{30} & \multirow{12}{*}{25} & \multirow{4}{*}{ I } & $\tilde{\alpha}$ & 0.1504 & 0.0894 & 0.0898 & 0.0893 & 0.0894 & 0.0883 \\
\hline & & & & $\widetilde{\beta}$ & 0.6422 & 0.5682 & 0.5687 & 0.5595 & 0.5682 & 0.5361 \\
\hline & & & & $\underset{\sim}{\widetilde{s}}(t)$ & 0.0152 & 0.0143 & 0.0144 & 0.0142 & 0.0143 & 0.0141 \\
\hline & & & & $\widetilde{h}(t)$ & 0.0041 & 0.0038 & 0.0039 & 0.0037 & 0.0038 & 0.0034 \\
\hline & & & \multirow{4}{*}{ II } & $\underset{\widetilde{\alpha}}{\alpha}$ & 0.1517 & 0.0926 & 0.0928 & 0.0925 & 0.0926 & 0.0923 \\
\hline & & & & $\widetilde{\beta}$ & 0.6521 & 0.5691 & 0.5695 & 0.5598 & 0.5691 & 0.5369 \\
\hline & & & & $\widetilde{\widetilde{s}}(t)$ & 0.0161 & 0.0146 & 0.0145 & 0.0143 & 0.0146 & 0.0142 \\
\hline & & & & $\tilde{h}(t)$ & 0.0047 & 0.0041 & 0.0042 & 0.0039 & 0.0041 & 0.0037 \\
\hline & & & \multirow{4}{*}{ III } & $\underset{\widetilde{\alpha}}{\alpha}$ & 0.1537 & 0.0954 & 0.0959 & 0.0951 & 0.0954 & 0.0936 \\
\hline & & & & $\tilde{\beta}$ & 0.6547 & 0.5695 & 0.5707 & 0.5617 & 0.5695 & 0.5487 \\
\hline & & & & $\widetilde{\widetilde{s}}(t)$ & 0.0166 & 0.0149 & 0.0152 & 0.0151 & 0.0149 & 0.0143 \\
\hline & & & & $\widetilde{h}(t)$ & 0.0052 & 0.0045 & 0.0047 & 0.0042 & 0.0045 & 0.0039 \\
\hline
\end{tabular}

TABLE 6: MSE of MLEs and BEs with true values.

\begin{tabular}{|c|c|c|c|c|c|c|c|c|c|c|}
\hline \multirow{3}{*}{$k$} & \multirow{3}{*}{$n$} & \multirow{3}{*}{$m$} & \multirow{3}{*}{ C.S } & & \multirow{3}{*}{$M L$} & \multirow{3}{*}{$B S$} & \multirow{2}{*}{\multicolumn{2}{|c|}{$\begin{array}{c}\mathrm{BL} \\
a\end{array}$}} & \multirow{2}{*}{\multicolumn{2}{|c|}{$\begin{array}{c}\text { BG } \\
q\end{array}$}} \\
\hline & & & & & & & & & & \\
\hline & & & & & & & -2 & 2 & -2 & 2 \\
\hline \multirow{12}{*}{1} & \multirow{12}{*}{50} & \multirow{12}{*}{30} & \multirow{4}{*}{ I } & $\tilde{\alpha}$ & 0.0914 & 0.0794 & 0.0834 & 0.0756 & 0.0838 & 0.0674 \\
\hline & & & & $\widetilde{\beta}$ & 0.6538 & 0.4130 & 0.4871 & 0.3425 & 0.4389 & 0.3352 \\
\hline & & & & $\tilde{\widetilde{s}}(t)$ & 0.0242 & 0.0222 & 0.0222 & 0.0222 & 0.0222 & 0.0222 \\
\hline & & & & $\tilde{h}(t)$ & 0.0054 & 0.0038 & 0.0037 & 0.0038 & 0.0037 & 0.0036 \\
\hline & & & \multirow{4}{*}{ II } & $\tilde{\alpha}$ & 0.0942 & 0.0798 & 0.0879 & 0.0788 & 0.0883 & 0.0686 \\
\hline & & & & $\tilde{\beta}$ & 0.6541 & 0.4140 & 0.4878 & 0.3450 & 0.4397 & 0.3379 \\
\hline & & & & $\tilde{\tilde{s}}(t)$ & 0.0255 & 0.0231 & 0.0231 & 0.0231 & 0.0231 & 0.0231 \\
\hline & & & & $\tilde{h}(t)$ & 0.0058 & 0.0039 & 0.0040 & 0.0038 & 0.0041 & 0.0037 \\
\hline & & & \multirow{4}{*}{ III } & $\tilde{\alpha}$ & 0.0945 & 0.0806 & 0.0888 & 0.0796 & 0.0891 & 0.0708 \\
\hline & & & & $\widetilde{\beta}$ & 0.6558 & 0.4304 & 0.5052 & 0.3575 & 0.4566 & 0.3503 \\
\hline & & & & $\tilde{\boldsymbol{s}}(t)$ & 0.0279 & 0.0245 & 0.0245 & 0.0245 & 0.0245 & 0.0245 \\
\hline & & & & $\tilde{h}(t)$ & 0.0059 & 0.0041 & 0.0042 & 0.0040 & 0.0043 & 0.0039 \\
\hline \multirow{12}{*}{5} & \multirow{12}{*}{50} & \multirow{12}{*}{30} & \multirow{4}{*}{ I } & $\tilde{\alpha}$ & 0.0919 & 0.0812 & 0.0843 & 0.0771 & 0.0847 & 0.0698 \\
\hline & & & & $\tilde{\beta}$ & 0.6483 & 0.4175 & 0.4879 & 0.3633 & 0.4639 & 0.3560 \\
\hline & & & & $\widetilde{s}(t)$ & 0.0194 & 0.0182 & 0.0182 & 0.0182 & 0.0182 & 0.0182 \\
\hline & & & & $\tilde{h}(t)$ & 0.0053 & 0.0034 & 0.0035 & 0.0034 & 0.0035 & 0.0034 \\
\hline & & & \multirow{4}{*}{ II } & $\widetilde{\alpha}$ & 0.0923 & 0.0826 & 0.0861 & 0.0797 & 0.0887 & 0.0727 \\
\hline & & & & $\tilde{\beta}$ & 0.6499 & 0.4263 & 0.4917 & 0.3721 & 0.4927 & 0.3648 \\
\hline & & & & $\tilde{s}(t)$ & 0.0196 & 0.0184 & 0.0184 & 0.0184 & 0.0184 & 0.0184 \\
\hline & & & & $\widetilde{h}(t)$ & 0.0054 & 0.0037 & 0.0038 & 0.0037 & 0.0038 & 0.0035 \\
\hline & & & \multirow{4}{*}{ III } & $\tilde{\alpha}$ & 0.0941 & 0.0843 & 0.0877 & 0.0806 & 0.0891 & 0.0756 \\
\hline & & & & $\tilde{\beta}$ & 0.6512 & 0.4344 & 0.5042 & 0.3852 & 0.4988 & 0.3778 \\
\hline & & & & $\widetilde{s}(t)$ & 0.0198 & 0.0187 & 0.0187 & 0.0188 & 0.0187 & 0.0188 \\
\hline & & & & $\tilde{h}(t)$ & 0.0055 & 0.0038 & 0.0040 & 0.0039 & 0.0040 & 0.0037 \\
\hline
\end{tabular}

Pro-F-F-C sample from GPD with the parameters $(\alpha, \beta)=$ $(0.5,2),(0.3,1)$ is generated. The true values of $s(t)$ and $h(t)$ at time $t=0.4$ and 0.5 are evaluated to be $(s(t)=0.9129, h(t)=0.2083)$ and $(s(t)=0.8855, h(t)=$ $0.2)$. The performance of the resulting estimators of $\alpha, \beta$, $s(t)$, and $h(t)$ has been considered in terms of the mean squared error (MSE), which are computed, for $l=1,2,3,4$, $M=1000, \phi_{1}=\alpha, \phi_{2}=\beta, \phi_{3}=s(t)$, and $\phi_{4}=h(t)$ as MSE $=1 / M \sum_{j=1}^{M}\left(\hat{\phi}_{l}^{()^{2}}-\phi_{l}\right)^{2}$. These results were obtained using Mathematica ver. 13. Considering two different group sizes $k=1,5$ and the following censoring schemes,

Scheme I: $R_{1}=n-m$ and $R_{i}=0$ for $i \neq 1$

Scheme II: $R_{m+1 / 2}=n-m$ and $R_{i}=0$ for $i \neq m+1 / 2$ if $m$ odd, and $R_{m / 2}=n-m$ and $R_{i}=0$ for $i \neq m / 2$ if $m$ even

Scheme III: $R_{m}=n-m$ and $R_{i}=0$ for $i \neq m$
The results of MSE of estimates are reported in Tables 5 and 6.

\section{Conclusion}

The main aim of this article is to develop different methods to estimate the unknown quantities of the GPD based on a Pro-FF-C scheme, which was introduced by Wu and Kuş [30]. We applied the classical and the Bayesian inferential procedures for the unknown parameters and reliability measures. The ACIs have been derived based on the asymptotic normality of MLEs. Under the Bayesian approach, we obtained the BEs based on the SE, LINEX, and GE loss functions. Furthermore, we assumed the conjugate gamma prior for the shape parameter and discrete prior for the scale parameter. The exact confidence interval and exact confidence region for the estimators have been constructed based on pivotal quantities. A numerical 
example using a simulated data set has been studied to show the practicality of these proposed procedures. The performance of the different estimation methods is realized via a simulation study which is revealed in the following:

(1) The BEs based on SE, LINEX, and GE loss functions perform better than the MLEs, in terms of MSEs

(2) The BEs based on LINEX and GE loss functions when $a=1$ and 2 and $q=1$ and 2 perform better than BEs based on SE, in terms of MSEs

(3) The BEs based on the SE loss function perform better than BEs based on LINEX and GE loss functions when $a=-1$ and -2 and $q=-1$ and -2 , in terms of MSEs

(4) From Tables 5 and 6, for a fixed scheme, the MSE values of all estimates, a model's parameters, and the reliability measures decrease as $\mathrm{m} / n$ increases which is consistent with the statistical theory that the larger the sample size, the more accurate the estimate

(5) It can be seen from Tables 5 and 6 that the three CS methods vary in terms of preference and sometimes CS I is the best while at other times the CS II or III is the best in the sense of having smaller MSEs

(6) The MSEs for $\alpha$ and $\beta$ estimates based on the Pro-FF-C scheme with $k=5$ increase in those for P-typeII-C with $k=1$ while the MSEs for $s(t)$ and $h(t)$ estimates based on the Pro-F-F-C scheme with $k=5$ decrease in those for P-type-II-C with $k=1$

\section{Data Availability}

The data used are theoretically generated from the equations in the manuscript.

\section{Conflicts of Interest}

The authors declare that they have no conflicts of interest.

\section{Acknowledgments}

This research was supported by Taif University Researchers Supporting Project (number TURSP-2020/318), Taif University, Taif, Saudi Arabia.

\section{References}

[1] N. Balakrishnan and R. A. Sandhu, "A simple simulational algorithm for generating progressive type-II censored samples," The American Statistician, vol. 49, no. 2, pp. 229-230, 1995.

[2] N. Balakrishnan and R. A. Sandhu, "Best linear unbiased and maximum likelihood estimation for exponential distributions under general progressive Type-II censored samples," Sankhya Series B, vol. 58, no. 1, pp. 1-9, 1996.

[3] A. C. Cohen, "Progressively censored samples in life testing," Technometrics, vol. 5, no. 3, pp. 327-339, 1963.

[4] N. R. Mann, "Best linear invariant estimation for weibull parameters under progressive censoring," Technometrics, vol. 13, no. 3, pp. 521-533, 1971.
[5] H. K. T. Ng, "Parameter estimation for a modified weibull distribution, for progressively type-II censored samples," IEEE Transactions on Reliability, vol. 54, no. 3, pp. 374-380, 2005.

[6] N. Balakrishnan, N. Kannan, C. T. Lin, and H. K. T. Ng, "Point and interval estimation for Gaussian distribution, based on progressively type-II censored samples," IEEE Transactions on Reliability, vol. 52, no. 1, pp. 90-95, 2003.

[7] D. I. Gibbons and L. C. Vance, "Estimators for the 2-parameter weibull distribution with progressively censored samples," IEEE Transactions on Reliability, vol. R-32, no. 1, pp. 95-99, 1983.

[8] H.-K. Yuen and S.-K. Tse, "Parameters estimation for weibull distributed lifetimes under progressive censoring with random removeals," Journal of Statistical Computation and Simulation, vol. 55, no. 1-2, pp. 57-71, 1996.

[9] H. K. T. Ng, P. S. Chan, and N. Balakrishnan, "Estimation of parameters from progressively censored data using EM algorithm," Computational Statistics \& Data Analysis, vol. 39, no. 4, pp. 371-386, 2002.

[10] N. Balakrishnan, "Progressive censoring methodology: an appraisal,” Test, vol. 16, no. 2, pp. 211-259, 2007.

[11] A. A. Soliman, "Estimation of parameters of life from progressively censored data using Burr-XII model," IEEE Transactions on Reliability, vol. 54, no. 1, pp. 34-42, 2005.

[12] A. A. Soliman, "Estimations for Pareto model using general progressive censored data and asymmetric loss," Communications in Statistics - Theory and Methods, vol. 37, no. 9, pp. 1353-1370, 2008.

[13] M. T. Madi and M. Z. Raqab, "Bayesian inference for the generalized exponential distribution based on progressively censored data," Communications in Statistics - Theory and Methods, vol. 38, no. 12, pp. 2016-2029, 2009.

[14] M. A. W. Mahmoud, M. Moshref, N. M. Yhiea, and N. M. Mohamed, "Progressively censored data from the weibull gamma distribution moments and estimation," Journal of Statistics Applications \& Probability, vol. 3, no. 1, pp. 45-60, 2014.

[15] M. A. W. Mahmoud, R. M. El-Sagheer, A. A. Soliman, and A. H. Abd-Ellah, "Inferences of the lifetime performance index with Lomax distribution based on progressive type-II censored," Economic Quality Control, vol. 29, pp. 39-51, 2014.

[16] A. A. Soliman, A. H. Abd Ellah, N. A. Abou-Elheggag, and R. M. El-Sagheer, "Inferences using type-II progressively censored data with binomial removals," Arabian Journal of Mathematics, vol. 4, no. 2, pp. 127-139, 2015.

[17] R. M. El-Sagheer, "Estimation using progressively Type-II censored data from Rayleigh distribution with binomial removals: Bayesian and non-Bayesian approach," JP J. Fund. Appl. Stat.vol. 8, no. 1, pp. 17-39, 2015.

[18] R. M. El-Sagheer, "Estimation of parameters of WeibullGamma distribution based on progressively censored data," Statistical Papers, vol. 59, no. 2, pp. 725-757, 2018.

[19] R. M. El-Sagheer, "Estimating the parameters of Kumaraswamy distribution using progressively censored data," Journal of Testing and Evaluation, vol. 47, no. 2, pp. 905-926, 2019.

[20] M. A. W. Mahmoud, R. M. El-Sagheer, and S. H. M. Abdallah, "Inferences for new Weibull-Pareto distribution based on progressively Type-II censored data," Journal of Statistics Applications \& Probability, vol. 5, no. 3, pp. 501-514, 2016.

[21] R. M. El-Sagheer and M. M. Hasaballah, "Inference of process capability Index Cpy for 3-Burr-XII distribution based on progressive Type-II censoring," International Journal of 
Mathematics and Mathematical Sciences, vol. 2020, pp. 1-13, 2020.

[22] R. M. El-Sagheer, E. M. Shokr, M. A. W. Mahmoud, and B. S. El-Desouky, "Inferences for weibull fréchet distribution using a bayesian and non-bayesian methods on gastric cancer survival times," Computational and Mathematical Methods in Medicine, vol. 2021, pp. 1-12, 2021.

[23] A. A. Soliman, E. A. Ahmed, A. H. Abd Ellah, and A. A. Farghal, "Bayesian estimation from exponentiated Frechet model using MCMC approach based on progressive Type-II censoring data," Statistics Applications \& Probability, vol. 4, no. 3, pp. 387-403, 2015.

[24] Y. Zhang and W. Gui, "Statistical inference for the lifetime performance index of products with Pareto distribution on basis of general progressive type II censored sample," Communications in Statistics - Theory and Methods, vol. 50, no. 16, pp. 3790-3808, 2021.

[25] R. Viveros and N. Balakrishnan, "Interval estimation of parameters of life from progressively censored data," Technometrics, vol. 36, no. 1, pp. 84-91, 1994.

[26] J.-W. Wu and H.-Y. Yu, "Statistical inference about the shape parameter of the Burr type XII distribution under the failurecensored sampling plan," Applied Mathematics and Computation, vol. 163, no. 1, pp. 443-482, 2005.

[27] J.-W. Wu, W.-L. Hung, and C.-H. Tsai, "Estimation of the parameters of the Gompertz distribution under the first failure-censored sampling plan," Statistics, vol. 37, no. 6, pp. 517-525, 2003.

[28] W.-C. Lee, J.-W. Wu, and H.-Y. Yu, "Statistical inference about the shape parameter of the bathtub-shaped distribution under the failure-censored sampling plan," International Journal on Information and Management Sciences, vol. 18, pp. 157-172, 2007.

[29] J.-W. Wu, T.-R. Liang-Yuh Ouyang, and L.-Y. Ouyang, "Limited failure-censored life test for the Weibull distribution," IEEE Transactions on Reliability, vol. 50, no. 1, pp. 107-111, 2001.

[30] S.-J. Wu and C. Kuş, "On estimation based on progressive first-failure-censored sampling," Computational Statistics \& Data Analysis, vol. 53, no. 10, pp. 3659-3670, 2009.

[31] A. A. Soliman, A. H. A. Ellah, N. A. Abou-Elheggag, and A. A. Modhesh, "Bayesian inference and prediction of Burr type XII distribution for progressive first failure censored sampling," Intelligent Information Management, vol. 03, no. 05, pp. 175-185, 2011.

[32] A. A. Soliman, A. H. Abd Ellah, N. A. Abou-Elheggag, and A. A. Modhesh, "Estimation of the coefficient of variation for non-normal model using progressive first-failure-censoring data," Journal of Applied Statistics, vol. 39, no. 12, pp. 2741-2758, 2012.

[33] A. A. Soliman, A. H. Abd-Ellah, N. A. Abou-Elheggag, and G. A. Abd-Elmougod, "Estimation of the parameters of life for Gompertz distribution using progressive first-failure censored data," Computational Statistics \& Data Analysis, vol. 56, no. 8, pp. 2471-2485, 2012.

[34] M. A. W. Mahmoud, A. A. Soliman, A. H. Abd-Ellah, and R. M. El-Sagheer, "Bayesian inference and prediction using progressive first-failure censored from generalized Pareto distribution," Journal of Statistics Applications \& Probability, vol. 2, no. 3, pp. 269-279, 2013.

[35] T. A. Abushal, "Estimation of the unknown parameters for the Compound Rayleigh distribution based on progressive firstfailure-censored sampling," Open Journal of Statistics, vol. 01, no. 03, pp. 161-171, 2011.
[36] E. A. Ahmed, "Estimation and prediction for the generalized inverted exponential distribution based on progressively firstfailure-censored data with application," Journal of Applied Statistics, vol. 44, no. 9, pp. 1576-1608, 2017.

[37] M. M. Mahmoud, M. M. Nassar, and M. A. Aefa, "Bayesian estimation and prediction based on progressively first failure censored scheme from a mixture of Weibull and Lomax distributions," Pakistan Journal of Statistics and Operation Research, vol. 16, no. 2, pp. 357-372, 2020.

[38] A. A.-E. Soliman, E. A. Ahmed, A. H. Abd Ellah, and A. A. Farghal, "Assessing the lifetime performance index using exponentiated Frechet distribution with the progressive first-failure-censoring scheme," American Journal of Theoretical and Applied Statistics, vol. 3, no. 6, pp. 167-176, 2014.

[39] Y. Cai and W. Gui, "Classical and Bayesian inference for a progressive first-failure censored left-truncated normal distribution," Symmetry Plus, vol. 13, no. 3, p. 490, 2021.

[40] E. Kremer, "A characterization of the generalized Paretodistribution with an application to reinsurance," Blätter der DGVFM, vol. 23, no. 1, pp. 17-19, 1997.

[41] D. R. Thomas and W. M. Wilson, "Linear order statistic estimation for the two-parameter weibull and extreme-value distributions from type II progressively censored samples," Technometrics, vol. 14, no. 3, pp. 679-691, 1972.

[42] S.-F. Wu, C.-C. Wu, Y.-L. Chen, Y.-R. Yu, and Y. P. Lin, "Interval estimation of a two-parameter Burr-XII distribution under progressive censoring," Statistics, vol. 44, no. 1, pp. 77-88, 2010. 